\title{
Use of parallel computing and visualisation techniques in the simulation of large scale geoenvironmental engineering problems
}

\author{
P.J. Cleall, H.R. Thomas, T.A. Melhuish and D.H. Owen \\ Geoenvironmental Research Centre, \\ Cardiff School of Engineering, Cardiff University, \\ PO Box 925, Cardiff, Cardiff, CF24 0YF
}

\begin{abstract}
This paper discusses some of the computational and visualisation challenges encountered in the field of geoenvironmental engineering. The use of iterative solvers and parallel computation methods are identified as being of particular relevance to addressing the computation challenges. 3D stereoscopic visualisation is discussed in relation to data analysis and communication. An example analysis is presented related to the issue of nuclear waste disposal where the use of iterative solvers and parallel algorithms was found to yield significant results.
\end{abstract}

Keywords: Parallel computing, 3D visualisation, geoenvironmental, geoscience 


\section{INTRODUCTION}

The field of geoenvironmental engineering has emerged from the merger of environmental issues, studies and concerns with traditional geotechnical engineering concepts. The development of this discipline is driven by a number of major environmental concerns including contaminated land, waste disposal, landfills, waste minimisation and underwater geoenvironmental issues. The problems encountered in this area are often complex in respect to both the processes occurring and their geometry and scale.

It is often of value to undertaken numerical analyses of these problems as this can lead to i) a clearer understanding of the processes involved, ii) predictions of future behaviour and iii) the development of sustainable solutions. In recent years the development of theoretical and numerical models for the types of processes occurring in these problems has had significant attention, with many models addressing highly non-linear coupled behaviour.

As the scale and complexity of problems considered has developed the computational demands of the work have increased. This has led to the implementation of high performance computing techniques, including parallel computing approaches and iterative solvers. Also, since the geometry and scale of problems has become more complex the use of sophisticated 3D visualisation techniques is now required. In this paper some of the computation demands encountered in the area of geoenvironmental engineering are discussed. A typical numerical formulation is presented and applied to an example simulation. The objective of this paper is to describe the type of computational challenges encountered in the analysis of geoenvironmental problems and to present some solution methods used to address these problems. 


\section{COMPUTATIONAL DEMANDS}

Geoenvironmental engineering problems often lead to very computationally demanding analyses. The nature of the problems can be extremely varied, however the computational demands are generally driven by three key areas, namely; $i$ ) domain size, ii) timescale and iii) complexity of analysis. Each of these areas is discussed in more detail in the following sections. In these sections a topical geoenvironmental problem of major international importance is used as an example for illustration. For clarity a brief description of the problem is presented below.

Deep geological repositories have been proposed for the disposal of high level nuclear waste. Analysis of the long term behaviour of the repositories is required so that an assessment of their performance can be made. There are a number of proposed configurations for such repositories where waste canisters are placed within a multibarrier system to control the migration of the radionuclides. These barriers include the canister itself, an engineered clay barrier surrounding the canister and the host material (often rock).

\subsection{Domain}

Obviously the size, type and resolution of domain required for a particular analysis has a direct bearing on the computation demands that an analysis will have. These demands relate to those affected by the computation size of the problem, in particular i) memory storage requirements and ii) computational requirements related to solution of large sets of simultaneous equations.

Analysis of real problems can lead to large complex three-dimensional domains. To overcome the computational demands of such problems simplifying assumptions are often made with regard to the geometry so as to reduce the size of the problem. However for many real problems such assumptions are not defendable and may lead to features of the systems behaviour being neglected or significant errors. The resolution of the analysis is 
also of importance. It is necessary to ensure that the domain used has a suitable level of resolution to ensure that the phenomena under consideration are adequately captured.

The analysis of geological waste disposal repositories mentioned above is a problem that clearly illustrates these issues. The geometry of the proposed repositories generally includes a number of tunnels or emplacement rooms with a number of canisters placed in each room. The nature of this geometry leads to a problem that requires a full three-dimensional analysis to be performed. The analysis requires the inclusion of the influence of the surrounding host rock to capture the overall groundwater response. It is therefore necessary for the domain to extend some hundreds of meters in each direction, thereby leading to a large overall domain size.

The resolution required is an important factor as analysis of large domains with a coarse spatial discretisation does not necessarily lead to high computational demands. However in this problem analysis of the performance of each barrier is required, this leads to a fine resolution in the region of the engineered barrier so that the thermal and hydraulic gradients developed can be correctly captured. The combination of $i$ ) the overall size, $i i)$ the three dimensional nature and $\mathrm{iii}$ ) the required resolution of the problem leads, for a finite element analysis, to a large scale detailed mesh.

\subsection{Timescale}

For many geoenvironmental problems steady state conditions often are either not achieved or do not represent the critical state of the system. In such cases it is necessary to consider the transient behaviour of the system. To achieve this time stepping procedures are often used, this allows the transient response of the system to be simulated. Depending on the timescale to be considered and the rate of change in the system this can lead to significant computational demands. These demands relate mainly to the computational requirements of the repeated solution of large sets of simultaneous equations. Disk storage of databases of 
results is also an issue in such analyses, however this can generally be overcome via judicious selection of output times and variables.

The analysis of geological waste disposal repositories again clearly illustrates these issues. In reality true steady state conditions will not occur in such a problem, as in the long term, (i.e. 100,000 years), the materials will be subject to mineral alteration and the far field boundary conditions will be affected by global changes, for example potential climate changes. Furthermore the short term response of the system is critical, for example the peak temperature reached is of importance and will occur in the short term as the heat energy production of the waste reduces with time. It is therefore necessary to perform a transient analysis over a long time scale.

Again the issue of resolution is critical; in this case, however, it is the temporal resolution of the analysis that affects the computational demands of the problem. For the repository problem it is initially necessary to capture the rapid development of the thermal field, requiring a fine temporal resolution, and to then simulate the slower hydraulic processes. This leads to the use of a variable temporal resolution, starting initially with a fine resolution and evolving to a coarser resolution as the analysis proceeds. For an analysis of 100,000 years this can lead to the solution of over 10,000 time steps being required.

\subsection{Complexity of analysis}

The complexity of an analysis will be affected by a number of factors; $i$ ) number of variables considered, $i$ ) level of coupling between variables, iii) non-linearity of system and iv) number of processes considered. Each of these factors will influence the computational demands of the problem.

The number of variables considered will directly affect the memory storage requirements and the computational requirements related to solution of large sets of simultaneous equations. The level of coupling between the variables will dictate how coupled a solution is required. For example a highly coupled problem will require a fully coupled solution. For 
non-linear systems iterative solution methods are often employed to obtain sufficiently accurate results. This leads to an increase in the required number of solutions of the simultaneous equations thereby increasing the computational requirements as well as the requirement of recalculation of the system equations.

The complexity of the analysis required to assess the performance of geological waste disposal repositories clearly illustrates these issues. In this problem a number of fields of behaviour require consideration including the thermal, hydraulic, gas, chemical and stressstrain fields. The response of each of these fields is highly non-linear and coupled to the other fields. Therefore a fully coupled non-linear analysis is required. Additionally consideration of the geochemistry of the system leads to the inclusion of a large number of additional processes. Often this is achieved via the use of a separate geochemical model which is coupled to the system equations in a stepwise fashion.

\section{DISCRETISATION TECHNIQUES}

The numerical solution of the theoretical models commonly used in geoenvironmental problems is often achieved by a combination of numerical discretisation techniques. For the example presented in this paper the finite element method is employed for the spatial discretisation and a finite difference time stepping scheme for temporal discretisation.

In particular the Galerkin weighted residual method is used to formulate the finite element discretisation. An implicit mid-interval backward difference algorithm is implemented to achieve temporal discretisation, since it has been found to provide a stable solution for highly non-linear problems [13]. With appropriate initial and boundary conditions the set of, typically non-linear, coupled governing differential equations can be solved. 


\subsection{Computer code}

A computer code, known as COMPASS (COde for Modelling PArtly Saturated Soil), has been developed to implement the numerical approach discussed above $[15,17]$. This includes an 'engine' written in FORTRAN 90 and a graphic user interface written in VISUAL BASIC. The engine includes an extensive library of finite elements and allows both steady state and transient 1,2 and 3D problems to be analysed. An extensive verification and validation program of COMPASS has been undertaken [15]. It is recognised, however, that validation of the code is a process rather than a series of events and as such work on this aspect continues.

\section{PARALLEL COMPUTING AND ITERATIVE SOLVERS}

The computational demands of many geoenvironmental problems, discussed in section 2 , have led to the use of high performance parallel computing (HPPC) and iterative solver techniques. The coupled non-linear non-symmetric nature of the problems under consideration requires very careful application of HPPC techniques to ensure that correct and efficient solutions are obtained.

HPPC techniques have been applied to two main areas of the numerical solution namely i) system equation construction and ii) system equation solution. In some cases both areas have been addressed whilst in others only one has been tackled [10, 16]. Two main techniques have been applied, namely i) iterative solvers and ii) sub structuring algorithms. In the following sections each of these techniques is discussed in more detail.

\subsection{Iterative solvers}

In recent years iterative solvers have become increasingly popular for the solution of numerical analysis systems. Such solvers have been found to be competitive, in comparison with direct solvers, for large and complex problems [11]. In particular, the Conjugate gradient algorithm has been found to be one of the most effective iterative methods for the 
solution of large systems of linear equations with sparse symmetric positive definite matrices [1].

Conjugate gradient methods often suffer from ill conditioning and so in many cases preconditioner techniques have been applied to improve convergence to an accurate solution [8]. However, the application of preconditioning will generally lead to some additional computational demand [2]. Therefore care must be taken to ensure that the use of preconditioners leads to an overall improvement in efficiency.

Preconditioned iterative solution algorithms [2] for the type of coupled geoenvironmental problems discussed earlier have been developed [10]. In this work parallel asymmetric compatible variants of the conjugate gradient, the Bi-Conjugate Gradient (Bi-CG), conjugate gradient squared (CGS) and Bi-Conjugate Gradient stabilised (Bi-CG STAB) methods have been implemented for non-linear fully coupled problems. Use of such variants is necessary because of the non-symmetric nature of the coupled governing equations considered. Preconditioning was performed via Jacobi, or ILU decomposition based on a Crout factorisation with an incompleteness of fill-in degree zero [i.e. ILU(0)]. To minimise memory requirements a compressed row storage scheme (CRS) has been utilised.

Since it is very desirable to speed up computations as much as possible, implementation of parallelisation techniques has also been explored. To this end efforts have been concentrated on the use of iterative solvers to parallelise the assembly and solution aspects. In particular parallel applications of the preconditioned iterative solvers discussed above have been developed [10].

The nature of the finite element discretisation makes distribution of the assembly and storage of the stiffness matrix between processors a fairly simple task. The system matrix can be divided by assigning a set of nodes to each processor. This has the advantage that it can be employed on both regular and irregular grids as the nodal assignment is independent of geometry. Global assembly of the stiffness matrix is only ever required for full ILU 
preconditioning, for each of the other preconditioners each processor assembles and stores local sub-matrices only.

In the work presented by Owen [10] a high level of parallel efficiency was achieved. The level of parallelisation of the preconditioning algorithms however affected the comparison. The ILU preconditioning algorithms implemented had no parallel capability with parallel gains delivered being solely due to the parallelisation of solution algorithms alone.

\subsection{Domain decomposition}

Domain decomposition approaches are an ideal route to take advantage of HPPC. For example this can be achieved via the implementation of sub-structuring techniques. Such sub structuring methodologies for multi-processes architectures stem from the widely developed paradigm termed divide and conquer [6, 7]. Farhat and Lesoinne [6] commented that many proposed computational methods for solving differential equations in multiprocessor architectures are of this style, and involve some form of domain decomposition.

For example sub-structuring has been successfully implemented in the finite element method by many authors in a variety of fields $[3,4,7,9]$. In the area of geoenvironmental engineering sub structuring algorithms have been developed which apply direct solvers within a multi-level parallel strategy $[16,18]$. Such approaches have been found to impact, in a positive way, on both computer storage requirement and computational time.

\section{3D VISUALISATION}

The analysis of large scale geoenvironmental problems inevitably leads to large complex data sets. These data sets often cover both large spatial and temporal domains and include

numerous variables. A particular feature of the data sets is their multiple dimensionality, with transient three dimensional data being very common. 
In addition to the use of sophisticated contour plotting and animation techniques the use of stereoscopic projection has recently been applied to geoenvironmental problems. The use of $3 \mathrm{D}$ visualisation techniques can significantly aid the interrogation and communication of such data. Such data visualisation allows the processes occurring within the system under consideration to be directly identified. Furthermore the actual physical geometry of the problem can be viewed interactively.

To perform this type of visualisation a stereoscopic capable projector or monitor is required in addition to a computation server. For example, a 3D visualisation facility has recently been established at the Geoenvironmental Research Centre, Cardiff University (the home institute of the authors). This facility consists of a front projected active stereoscopic display with 2000 lumen digital projector driven by a $2.4 \mathrm{GHz}$ dual processor PC. This facility was relatively low cost at approximately £95K (160K US\$).

Such systems are very valuable in geoenvironmental applications due to the need to communicate effectively both the problem and any proposed solution. Such communication can occur at a number of levels, including: i) technical analysis ii) explanation to regulatory bodies and iii) public consultation. Although each of these levels of communication has its own particular needs, for all the ability to clearly communicate the behaviour of a complex system in a simple manner is the key benefit of such stereoscopic visualisation.

\section{THEORETICAL FORMULATIONS EMPLOYED}

For the many geoenvironmental problems a description of the coupled flow and deformation behaviour of soils is required. An example is the coupled thermal-hydraulic-mechanical response of a soil subject to various loading. In this case a three phase porous material consisting of solid, liquid and gas requires consideration. A set of coupled governing differential equations are presented below to describe heat and moisture flow coupled with the stress-strain response of the soil. The primary variables of the model are pore water 
pressure, $u_{l}$, temperature, $T$ and displacement $\mathbf{u}$. The development of these equations is described in more detail in [14] and [15].

\subsection{Heat transfer}

Conservation of heat energy can be defined via a classical conservation equation.

$$
\frac{\partial \Omega_{H}}{\partial t}=-\nabla \cdot Q
$$

where the heat content of unsaturated soil per unit volume, $\Omega_{H}$, includes latent heat of vapourisation and the classical components of heat capacity and $Q$ the heat flux per unit area.

\subsection{Moisture transfer}

The governing equation for moisture transfer in an unsaturated soil can be expressed as

$$
\begin{aligned}
\frac{\partial\left(\rho_{l} n S_{l}\right)}{\partial t}+\frac{\partial\left(\rho_{v} n\left(S_{l}-1\right)\right)}{\partial t} & =-\rho_{l} \nabla \cdot \mathbf{v}_{\mathbf{l}}-\rho_{l} \nabla \cdot \mathbf{v}_{\mathbf{v}} \\
& -\nabla \cdot \rho_{v} \mathbf{v}_{\mathbf{a}}
\end{aligned}
$$

where the velocities of pore liquid, $\mathbf{v}_{\mathbf{l}}$, and pore air, $\mathbf{v}_{\mathbf{a}}$, are based on a generalised Darcy's law, $\mathrm{n}$ is the porosity, $\rho_{l}$ is the liquid density, $\rho_{v}$ is the vapour density and $S_{l}$ is the degree of saturation. The definition of vapour velocity follows the approach presented by Thomas and King [13] and is based on vapour movement due to gradients of relative humidity.

\subsection{Constitutive stress-strain relationship}

For problems in unsaturated clays the total strain, $\varepsilon$, is assumed to consist of components due to suction, temperature and stress changes. This can be given in an incremental form, without loss of generality, as

$$
d \varepsilon=d \varepsilon_{\sigma}+d \varepsilon_{s}+d \varepsilon_{T}
$$

where the subscripts $\sigma, \mathrm{T}$ and $\mathrm{s}$ refer to net stress, temperature and suction contributions.

The stress strain relationship can therefore be expressed as 


$$
d \sigma^{\prime \prime}=\mathbf{D}\left(d \underline{\varepsilon}-d \underline{\varepsilon}_{s}-d \underline{\varepsilon}_{T}\right)
$$

where $\sigma^{\prime \prime}$ is the net stress and $\boldsymbol{D}$ is the elastic matrix. A number of constitutive relationships can be employed, for example an elasto-plastic constitutive relationship [15].

\subsection{Coupled equations}

This leads to a series of governing differential equations which can be expressed in terms of the primary variables of the model as $[14,15]$

Energy balance:

$$
C_{T l} \frac{\partial u_{l}}{\partial t}+C_{T l} \frac{\partial T}{\partial t}+C_{T u} \frac{\partial \mathrm{u}}{\partial t}=\nabla\left[K_{T l} \nabla u_{l}\right]+\nabla\left[K_{T T} \nabla T\right]+V_{T l} \nabla u_{l}+V_{T T} \nabla T+J_{T}
$$

Mass balance:

$$
C_{l l} \frac{\partial u_{l}}{\partial t}+C_{l T} \frac{\partial T}{\partial t}+C_{l u} \frac{\partial \mathrm{u}}{\partial t}=\nabla\left[K_{l l} \nabla u_{l}\right]+\nabla\left[K_{l T} \nabla T\right]+J_{l}
$$

Stress equilibrium:

$$
C_{u l} d u_{l}+C_{u T} d T+C_{u u} d \mathrm{u}+d \mathrm{~b}=0
$$

where $K_{i j}$ and $C_{i j}$ represent the corresponding terms of the governing equations $(i, j=l, T, u)$.

\section{APPLICATION}

In this section the analysis of a geoenvironmental problem is described with a focus on the computational demands and data interrogation requirements. The problem is related to the issue of deep geological repositories that was briefly discussed in section 2. In particular the Prototype Repository test is considered. This is a full scale experiment currently being performed by SKB, at their Hard Rock Laboratory in Aspö [12].

The objective of this international, EC supported, activity is to investigate the integrated performance of engineered barriers and near-field rock of a simulated deep repository in 
crystalline rock with respect to heat evolution, water permeation, mechanics, water chemistry, gas evolution and microbial processes under natural and realistic conditions at $450 \mathrm{~m}$ depth below the ground surface. The test site is a $65 \mathrm{~m}$ long TBM-bored drift from which six $1.75 \mathrm{~m}$ diameter deposition holes extend downwards to about $8 \mathrm{~m}$ depth in accordance with the KBS-3 concept [12]. The deposition holes will contain copper/steel canisters with heaters for simulating the heat energy generated by spent fuel rods. The canisters will be embedded in a dense buffer clay and the tunnel backfilled with a mixture of clay and crushed rock. The computer model COMPASS, employing the theoretical formulation described in section 6 , has been used to analyse the system.

The complex geometry necessitates the use of a 3D domain to correctly simulate the processes occurring. Furthermore the coupled highly non-linear nature of the problem (for example the coupling between the thermal and hydraulic fields) requires a relatively fine mesh in the regions close to the canisters. The expected timescale of the experiment is approximately 20 years, however predictions of behaviour beyond this point are also of interest. For the reasons discussed in section 2 this is therefore a very demanding computational problem.

Figure 1 shows a geometrical model of the Prototype Repository. This model has been discretised with a finite element mesh comprised of 140,367 nodes and 129,316 8 noded brick elements. A thermal-hydraulic analysis of the tunnel has been undertaken. Both parallel computing and iterative solvers have been employed to enable the analysis of this problem. In particular an ILU-Crout preconditioner was used with an iterative Bi-Conjugate Gradient stabilised (Bi-CG-STAB) solver as developed by Owen [10]. This analysis was performed, on a SUN Enterprise E6500 server, in parallel on 4 processors and took an average of 1.5 hours total computational time to analyse one time step, (this analysis consisted of approximately 1000 time steps). In comparison, simulation of the same problem using a direct serial solver was prohibitively slow. The use of parallel computing 
techniques and iterative solvers was therefore a requirement of obtaining a solution of the problem.

Interrogation of the analysis of this system was performed on the $3 \mathrm{D}$ stereoscopic visualisation facility discussed in section 5. As an example, the thermal regime of the system at a time of 10 years is shown in figures 2 and 3 . Figure 2 shows the interaction of each of the canisters on the thermal field this clearly illustrates the need for a 3D analysis of the system. Figure 3 shows the interrogation of data via isotherms. Furthermore the visualisation facility allows the interactive interrogation of the data with the viewer being able to move within the data. This example illustrates how data visualisation can allow the development of the fields of behaviour of interest to be directly interpreted.

\section{CONCLUSIONS}

This paper has discussed some of the computational and visualisation challenges encountered in the field of geoenvironmental engineering. In relation to the computational demands the domain size, the timescale and the complexity of the problem have been identified as the key factors. Work performed in addressing some of the computational and visualisation challenges encountered in this context were discussed. The use of iterative solvers and parallel computation methods were identified as being of particular relevance to addressing the computation challenges, whilst 3D stereoscopic visualisation was discussed in relation to data analysis and communication.

Finally an example was presented, via use of typical discretisation methods and theoretical formulations, related to the issue of nuclear waste disposal. This example clearly illustrated the type of demands made by geoenvironmental problems. With a complex problem analysed over both a large domain and time scale. The use of iterative solvers and parallel algorithms was found to yield significant savings in processor time whilst 3D visualisation techniques were found to be valuable in the analysis of the data sets produced. 
Finally it can be concluded that whilst geoenvironmental problems pose some very significant challenges the use of HPPC, iterative solvers and visualisation techniques have been found to be very valuable in addressing a number of the key issues related to these challenges.

\section{ACKNOWLEDGEMENTS}

The collaboration with SKB on the Prototype Repository project is gratefully acknowledged. The work presented here has been partly funded by an EPSRC studentship and an EC grant (FIKW-2000-00055) this support is also gratefully acknowledged.

\section{REFERENCES}

[1] Axelsson O. (1985"A survey of preconditioned iterative methods for linear systems of algebraic equations", BIT, 25: 166-187

[2] Barrett, R., Berry, M., Chan, T., Demmel, J., Donato, J., Dongraa, L., Eijkhout, V., Pozo, R., Romine, C., and Van derVorst, H., (1995) "Templates, for the solution of linear systems: building blocks for iterative methods", John Wiley Press, New York

[3] Baugh, J.W. and Chadha, H. S., (1993) "Network distributed finite element analysis", Information for technology for civil and structural engineers, Civ-Comp Press ISBN 0-948749-16-4, 205-218.

[4] Bjorstad, P. E. Braekhus, J. and Hvidsten, A. (1990). "Parallel sub-structuring algorithms in structural analysis, direct and indirect methods", 4th International symposium on domain decomposition methods for partial differential equations, SIAM 321-340.

[5] Farhat, C. (1989). "Which parallel finite element algorithm for which architecture and which problem", Computational structural mechanics and multidisciplinary optimization, R.V. Gradhi et al (eds), AD-V16, ASME, New York 35-43.

[6] Farhat, C. and Lesoinne, M., (1993) "Automatic partitioning of unstructured meshes for the parallel solution of problems in computational mechanics", International Journal for Numerical Methods in Engineering, 36, 745-764

[7] Farhat, C. and Wilson, E. (1987). "A new finite element concurrent computer program architecture", International Journal for Numerical Methods in Engineering, 24, 1771-1792. 
[8] Judice and Patrico (1994) "Truncated envelope preconditioning technique" Communications numerical methods in engineering, 10, 149-154.

[9] Notay, I. (1995) “An efficient parallel discrete PDE solver” Parallel Computing, 21, 1725-1748.

[10] Owen, D.H. 2000. Preconditioned parallel iterative solution methods for coupled finite element analyses, Ph.D. Thesis, University of Wales, Cardiff, U.K.

[11] Pool E. L., Knight, N. R. and Davis, D. D. (1992), "High-performance equation solvers and their impact on finite element analysis" International journal for numerical methods in engineering, 33: 855-868.

[12] Svemar, C. \& Pusch, R. (2000). Prototype Repository - Project description. SKB IPR-00-30.

[13] Thomas, H. R. and King, S. D. (1991). "Coupled temperature/capillary variations in unsaturated soil." ASCE, Journal of Eng. Mech., 117(11), 2475-2491.

[14] Thomas, H. R. and He, Y.(1995) "Analysis of coupled heat, moisture and air transfer in a deformable unsaturated soil." Geotechnique, 45(4),677-689

[15] Thomas, H. R, He, Y and Onofrei, C, (1998). "An examination of the validation of a model of the hydro/thermo/mechanical behaviour of engineered clay barriers". Int.J.for Numerical and Analytical Methods in Geomechanics, $22(1), 49-71$.

[16] Thomas, H. R., Yang, H. T., He, Y. and Jefferson A. D., (1998), "Solving coupled thermo-hydromechanical problems in unsaturated soil using a substructuring Frontal technique". Communications in Numerical Methods in Engineering, 14, 783-792.

[17] Thomas, H. R. and Cleall, P.J (1999), "Inclusion of expansive clay behaviour in coupled thermo hydraulic mechanical models", Int. Jour. of Engineering Geology 54: 93-108, 1999

[18] Thomas, H R, Yang, H T, He, Y and Cleall, P.J.,. (2003) "A multi-level parallelised substructuring frontal solution for coupled thermo/hydro/mechanical problems in unsaturated soil". International Journal for Numerical and Analytical Methods in Geomechanics 27:951-965. ISSN no: 0363-9061 


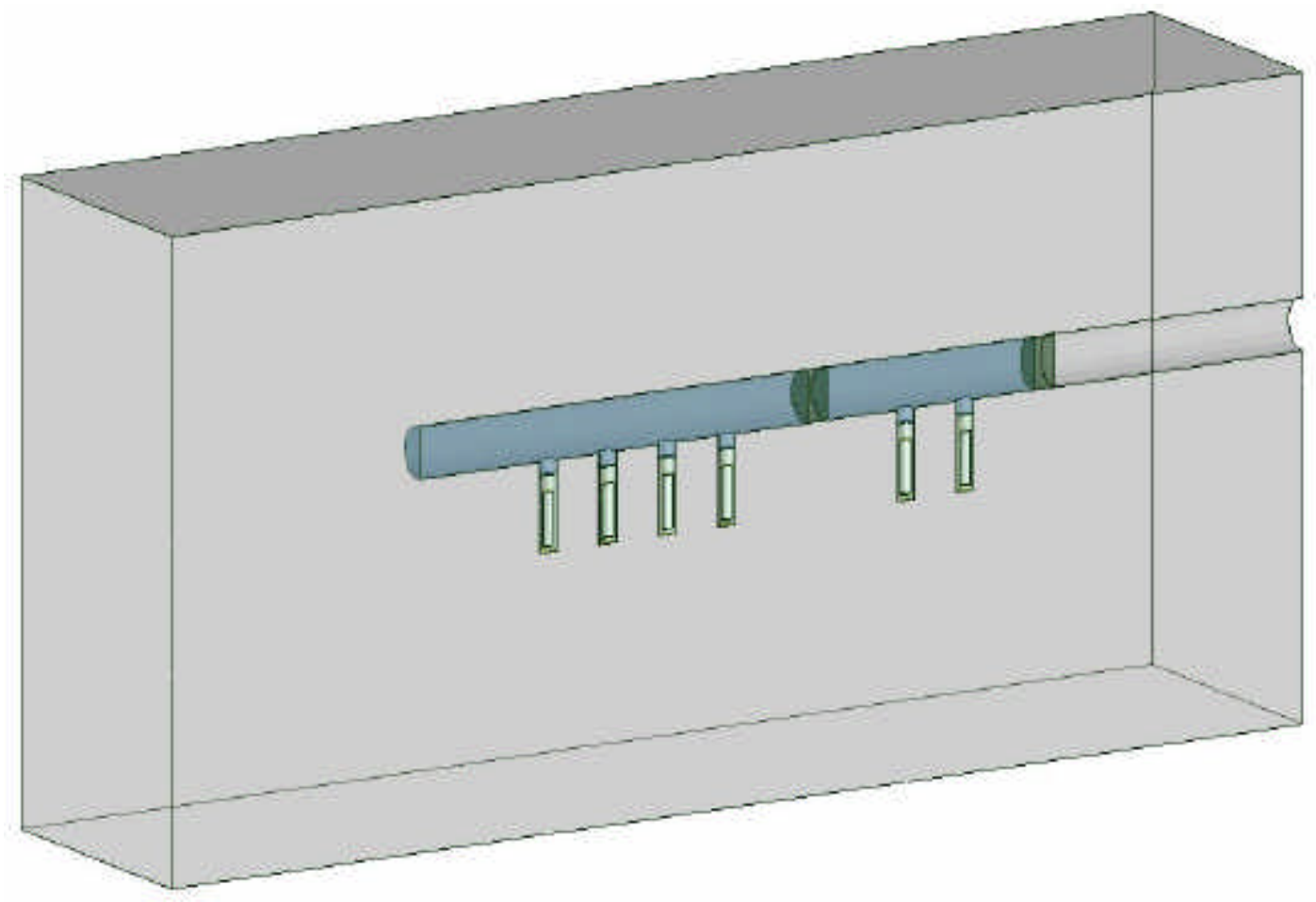

Figure 1 Geometric model of the Prototype Repository and deposition holes

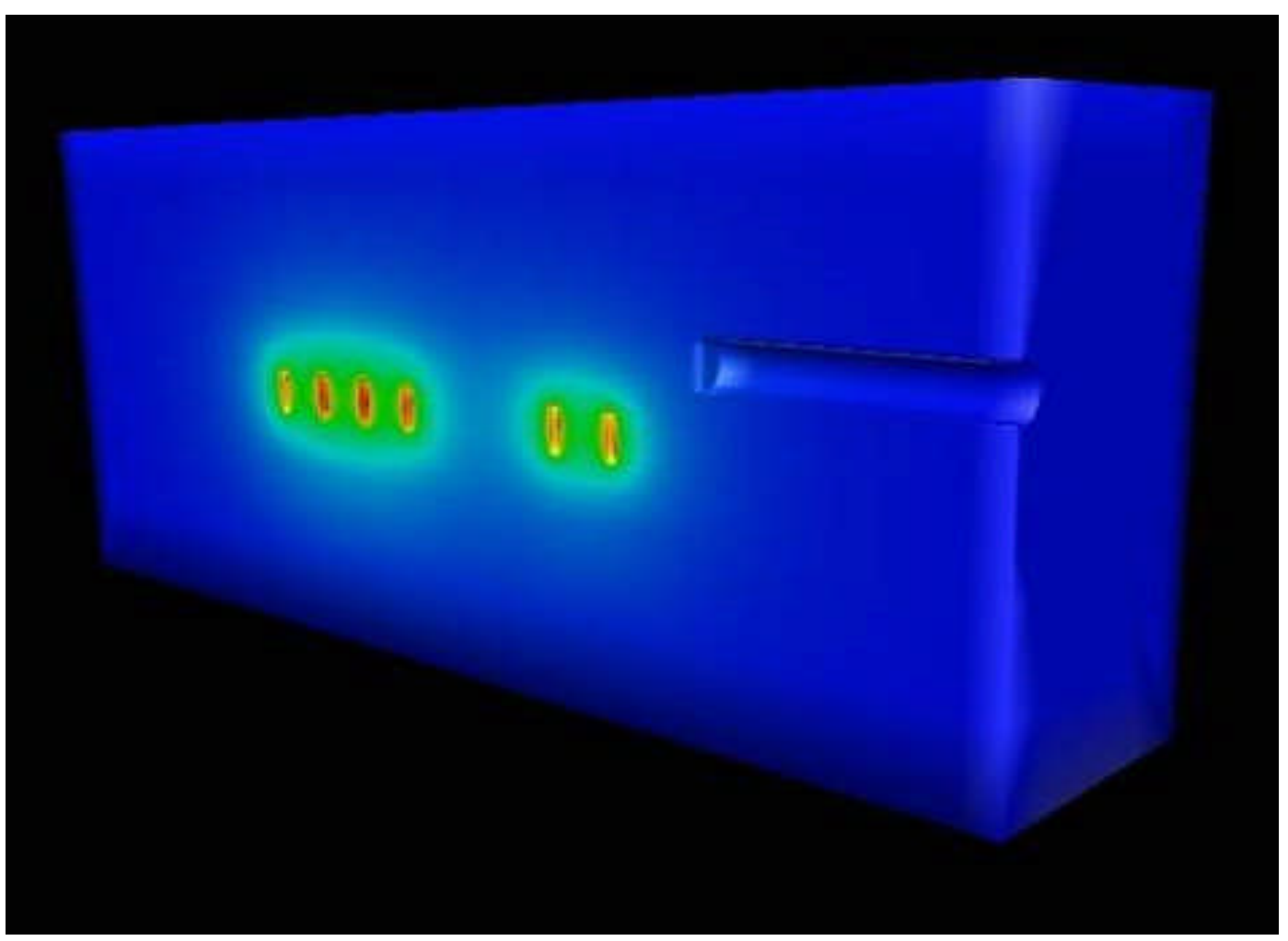

Figure 2 Temperature contours of the full Prototype Repository 


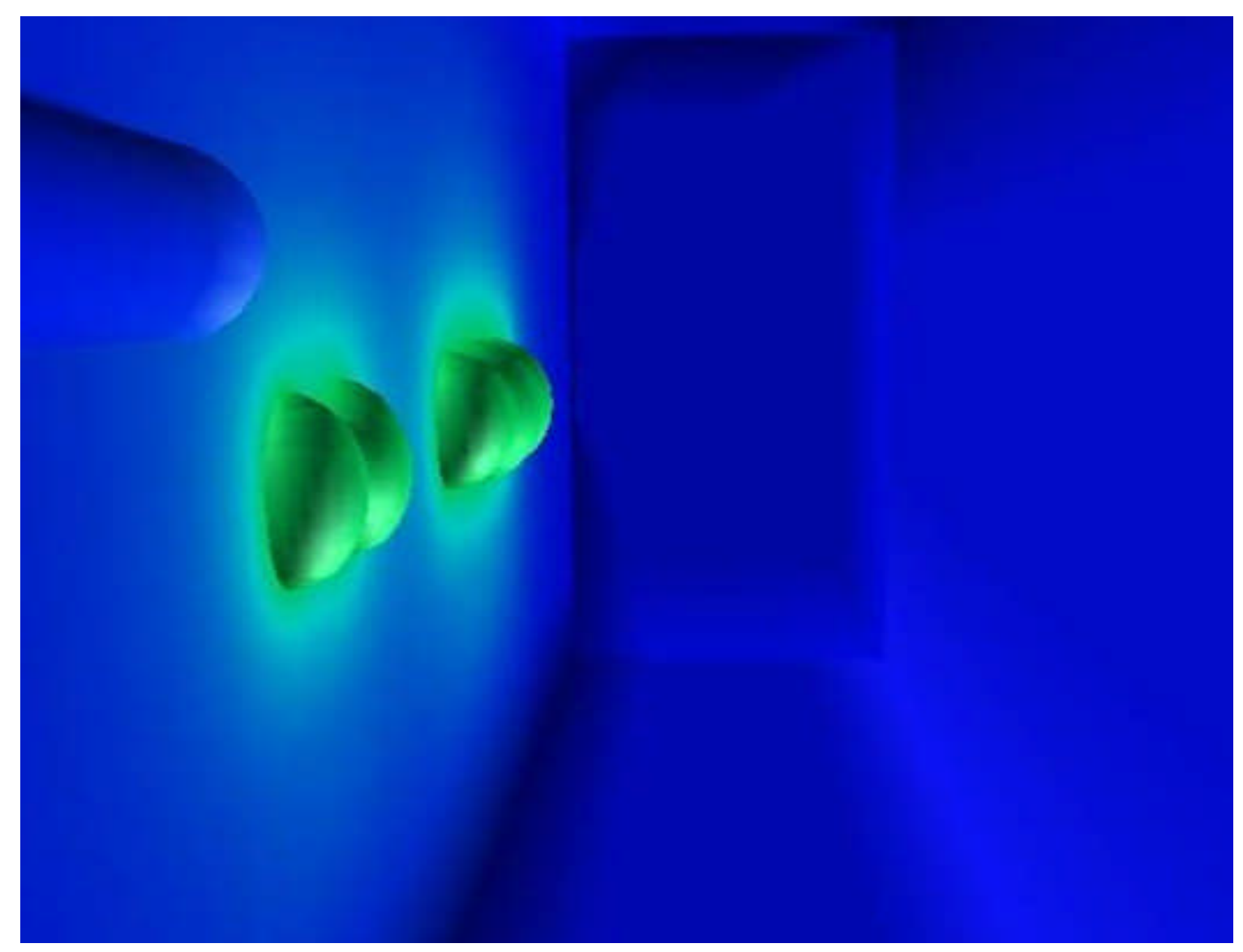

Figure 3 Isotherms of temperature viewed from inside the domain 